Ful I magnet ohydrodynami c si mul at $i$ on of the tilting instability in a field-reversed conf i gur at i on

\begin{tabular}{|l|l|}
\hline $\begin{array}{l}\text { jour nal or } \\
\text { publ i cat i on } \mathrm{titl} \text { e }\end{array}$ & Physi cs of Fl ui ds B \\
\hline vol une & Vol.1 \\
\hline number & No. 3 \\
\hline page range & pp. 581- 590 \\
\hline year & 1989- 03 01 \\
\hline URL & ht t p: //hdl . handl e. net /10655/1305 \\
\hline
\end{tabular}




\title{
Full magnetohydrodynamic simulation of the tilting instability in a field- reversed configuration
}

\author{
Ritoku Horiuchi \\ Science Project Corporation, Kuchita-Mimami 3-40-17-304, Asakita-ku, Hiroshima 739-19, Japan \\ Tetsuya Sato \\ Institute for Fusion Theory, Hiroshima University, Hiroshima 730, Japan
}

(Received 27 May 1988; accepted 15 November 1988)

\begin{abstract}
Nonlinear evolution of the tilting instability in a field-reversed configuration (FRC) is investigated by means of a three-dimensional full magnetohydrodynamic simulation. Three types of plasma model are considered, i.e., case (a) where the plasma is confined by a uniform external field, case (b) where the plasma is confined by a mirror external field, and case (c) where the plasma rotates around the major axis. For the prolate FRC, the internal tilt mode cannot stay at a low amplitude but keeps growing irrespective of whether the mirror field exists or not. When the initial configuration is substantially deformed, the instability triggers an external mode at the plasma-vacuum boundary because of the pressure imbalance. The outgoing flow driven by the external mode tears the plasma into two pieces, and the torn pieces move away toward the axial edges. The growth time is nearly equal to the transit time for the Alfvén wave to propagate over the plasma length. When the plasma is driven to spin with the Mach number of $M=1.5$, the growth rate is halved compared to the nonrotating case. The spin stabilization is expected to be complete for $M>2.5$. It is also found that the oblate FRC plasma is linearly unstable against the external tilt mode.
\end{abstract}

\section{INTRODUCTION}

The field-reversed configuration (FRC) is attractive in the sense that a high-beta plasma can be confined by a simple magnetic geometry. Linear analysis ${ }^{1-3}$ predicts that an elongated prolate FRC becomes unstable against the internal tilt mode. However, no experimental evidence ${ }^{4}$ has so far been reported on the tilt disruption in the magnetohydrodynamic (MHD) time scale predicted by the linear analysis. The explanation proposed by Barnes et al. ${ }^{5}$ and Ishida $\mathrm{et} \mathrm{al}^{6}$ is that the ion kinetic effect stabilizes the internal tilt mode when the spatial scale of the FRC plasma is comparable to the ion Larmor radius. If we extend the present device to reactor size, the device scale would be several hundred times larger than the ion Larmor radius. ${ }^{7}$ Then, the ion kinetic effect would be greatly reduced and the tilting instability would increase in power. One hope is that some nonlinear saturation mechanism could protect the FRC plasma from the destructive growth of the tilt mode.

In order to examine the nonlinear saturation effect, it would be best at present to investigate the nonlinear evolution of the tilt mode by means of a full MHD simulation. Moreover, the investigation may provide us with some information on a minimum energy state of the high-beta plasma.

There are two difficulties to be overcome in carrying out the simulation of the FRC plasma. The first difficulty is that one must deal with a system that contains both the plasma and the vacuum. The second one is how to treat a numerical singular point originating from the character of the chosen coordinate system. For example, the cylindrical coordinates $(r, \phi, z)$, which will be used in this paper, have a singular point at the central axis $r=0$. In order to overcome these difficulties we developed a new simulation code with fourth- order accuracy both in space and time, which is superior to the previously used code ${ }^{8}$ in clarifying the nonlinear evolution of the tilting instability.

The purpose of this paper is to investigate the nonlinear evolution of the tilt mode in a field-reversed configuration and clarify the mechanism leading to the tilting instability. In Sec. II we describe the fourth-order-accuracy simulation code and three types of simulation models. The results obtained from the simulation are discussed separately for each model in Sec. III. Finally we give a brief discussion in Sec. IV.

\section{NUMERICAL SCHEME AND SIMULATION MODEL}

Let us describe the numerical scheme that relies on the explicit finite-difference method with fourth-order accuracy both in space and time. The spatial derivative of a variable $F(x)$ is expressed in the central differencing form as

$$
\begin{aligned}
\left(\frac{d F(x)}{d x}\right)_{x=x(j)}= & \frac{1}{12 \Delta x}[-F(j+2)+8 F(j+1) \\
& -8 F(j-1)+F(j-2)],
\end{aligned}
$$

based on the Taylor expansion up to the fourth order of the grid separation $\Delta x$, where $j$ denotes the grid address along the spatial coordinate $x$. If we rewrite the spatial derivatives to the central differencing form by using the relation (1), we can deal with a set of partial differential equations as simultaneous ordinary differential equations in respect to time $t$. Then, we apply the fourth-order Runge-Kutta-Gill (RKG) method $^{9}$ in order to solve the equations and obtain the temporal evolution. The numerical error of this scheme can be estimated using a set of two linearized equations with plane wave solutions as $f_{p}(x, t)=f_{0} \exp (i k x-i \omega t)$ and 
$h_{p}(x, t)=-k / \omega f_{p}(x, t)$. If $f(x, t)$ and $h(x, t)$ satisfy the exact solutions $f_{p}(x, t)$ and $h_{p}(x, t)$ at $t=t_{0}$, the value of $f(x, t)$ at the next time step $t=t_{0}+\Delta t$ is given by

$$
f\left(x, t_{0}+\Delta t\right)=g(\alpha, \theta) f\left(x, t_{0}\right),
$$

where

$$
\begin{aligned}
& g(\alpha, \theta)=1+H+\frac{H^{2}}{2}+\frac{H^{3}}{6}+\frac{H^{4}}{24}, \\
& H=-i(\alpha / 3)(4-\cos 2 \theta) \sin 2 \theta \\
& \alpha=\omega \Delta t / k \Delta x, \quad \theta=k \Delta x / 2 .
\end{aligned}
$$

Setting $g(\alpha, \theta)$ equal to $|g| \exp (-2 i \alpha \theta-i \Delta \Phi)$, we can estimate the amplification factor $|g|$ and phase error $\Delta \Phi$. Figure 1 shows the $\alpha$ dependence of $|g|$ for $\theta=\pi / 4$ (top) and the $\theta$ dependence of $\Delta \Phi$ for $\alpha=0.8$ (bottom) at $t=t_{0}+8 k \Delta x / \omega$, where the results of the two-step LaxWendroff (LW) method ${ }^{10}$ are also shown for comparison. The fourth-order RKG scheme is more appropriate for numerical simulation for the following reasons. In order to obtain a physically reliable result it is necessary to carry out the simulation run under the condition that the amplification factor is always a little smaller than 1 . Figure 1 shows that the condition is satisfied in the wide range of $\alpha$ for the fourthorder RKG scheme while it is satisfied only in narrow regions for the two-step LW method, i.e., $0<\alpha<0.1$ and $0.9<\alpha<1.0$ (these regions are somewhat variable dependent on the wavelength we pay attention to). The operation of $0.9<\alpha<1.0$ is not appropriate to describe the nonlinear evolution because the system condition will change both in space and time and hence may happen to step in the numerically unstable region of $\alpha>1$ (Courant-Friedrichs-Lewy condition $\left.^{11}\right)$. For practical use the operation at $0<\alpha<0.1$ is needed for the two-step LW method. On the other hand, it is possible to carry out the fourth-order RKG scheme in the vicinity of $\alpha=1$. In other words, we can employ a time step
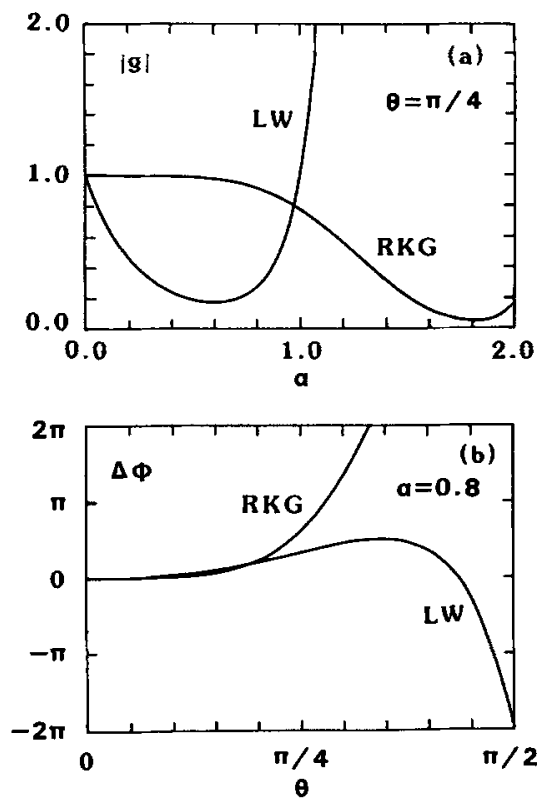

FIG. 1. The amplification factor $|g|$ for the case of $\theta=\pi / 4$ (top) and the phase error $\Delta \Phi$ for the case of $\alpha=0.8$ (bottom) at $t=t_{0}+8 k \Delta x / \omega$. The line RKG shows the result of the fourth-order Runge-Kutta-Gill scheme while the line LW shows the result of the two-step Lax-Wendroff scheme. somewhat larger than that of the two-step LW scheme. The leapfrog scheme ${ }^{10}$ has also been studied as an alternative method to reduce the numerical diffusion. ${ }^{12}$

The new scheme is found to be numerically stable if the condition $\alpha<1.5$ always holds for a periodic boundary problem. However, a numerical error accumulates against a gridsize mode for a bounded problem because the fourth-order central differencing cannot be applied to the spatial derivatives at the boundary and one mesh point from the boundary. That is, the four-point (not centered) differencing method is used at one mesh point from the boundary and the threepoint differencing method is used at the boundary in this paper. Fourth-order artificial smoothing technique is thus introduced to exclude the numerical error of the grid-size mode. The technique is as follows: let the value of a variable on the grid $j$ be denoted by $F(j)$. Then, the smoothing process is expressed by the replacement as

$$
F(j) \rightarrow F(j)\left(1-M_{f}\right)+M_{f}\langle F(j)\rangle,
$$

where $M_{f}=\frac{3}{8}$ and

$$
\begin{aligned}
\langle F(j)\rangle= & {[-F(j+2)+4 F(j+1)} \\
& +4 F(j-1)-F(j-2)] / 6
\end{aligned}
$$

is the fourth-order average value of $F(j)$. This replacement can be interpreted as the smoothing through a low-pass filter in the Fourier space. Figure 2 shows the filter functions of the fourth-order scheme (solid line) and the second-order scheme (dashed line) where the second-order smoothing is obtained by setting $\langle F(j)\rangle$ equal to the second-order average value $[F(j+1)+F(j-1)] / 2$ and $M_{f}$ to $\frac{1}{4}$ in Eq. (3). It is clear that this fourth-order smoothing keeps small wavenumber modes almost unchanged while it excludes large wavenumber modes more effectively compared with the second-order scheme.

Now we give the resistive full MHD equations in dimensionless units which are to be solved by means of the fourthorder scheme described above:

$$
\begin{aligned}
& \frac{\partial \rho}{\partial t}=-\boldsymbol{\nabla} \cdot(\rho \mathbf{v}), \\
& \rho \frac{\partial \mathbf{v}}{\partial t}=-\rho(\mathbf{v} \cdot \nabla) \mathbf{v}-\nabla p+\mathbf{j} \times \mathbf{B},
\end{aligned}
$$

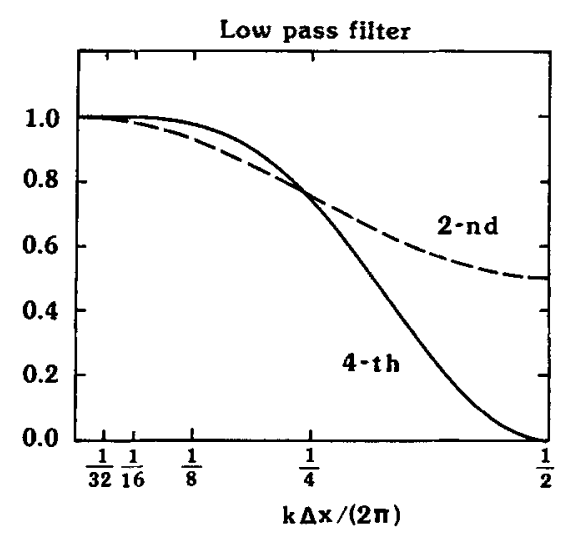

FIG. 2. Wavenumber dependence of the filter function for the second-order scheme (dashed line) and the fourth-order scheme (solid line). 


$$
\begin{aligned}
& \frac{\partial \mathbf{B}}{\partial t}=\nabla \times(\nabla \times \mathbf{B}-\eta \mathbf{j}), \\
& \frac{\partial U}{\partial t}=-\nabla \cdot \mathbf{g},
\end{aligned}
$$

where

$$
\begin{aligned}
& \mathbf{g}=[U+p+(\mathbf{B} \cdot \mathbf{B}) / 2] \mathbf{v}-(\mathbf{B} \cdot \mathbf{v}) \mathbf{B}+\eta \mathbf{j} \times \mathbf{B}, \\
& \mathbf{j}=\mathbf{\nabla} \times \mathbf{B}, \quad U=\rho(\mathbf{v} \cdot \mathbf{v}) / 2+\rho \epsilon+(\mathbf{B} \cdot \mathbf{B}) / 2,
\end{aligned}
$$

and $\epsilon$ is the internal energy per unit mass, $\boldsymbol{\eta}$ is the electrical resistivity, $p$ is the thermal pressure, $\rho$ is the matter density, and $v$ is the matter velocity. The equation of state is assumed to be

$$
\epsilon=p /[\rho(\gamma-1)],
$$

where $\gamma$ is the ratio of specific heats.

Since the tilt mode is essentially ideal and its time scale is given by the Alfvén transit time,$^{1-3}$ the resistive process does not play an important role. In this work, a uniform resistivity, which corresponds to the magnetic Reynolds number $S_{m}=10^{4}$, is assumed for simplicity. The initial pressure and density profiles are assumed to be

$$
\begin{aligned}
& p= \begin{cases}p_{0}\left[\left(\Phi_{0}-\Phi\right) /\left(\Phi_{0}-\Phi_{n 1}\right)\right]^{\sigma}, & \text { if } \Phi<\Phi_{0}, \\
0, & \text { if } \Phi \geqslant \Phi_{0},\end{cases} \\
& \rho=\rho_{0}\left(p / p_{0}\right)^{1 / \gamma},
\end{aligned}
$$

where $\Phi$ is the poloidal flux function and $\Phi_{\mathrm{nl}}(<0)$ is its value on the field null point (the " 0 " point of a magnetic field line); $\sigma, p_{0}, \rho_{0}$, and $\Phi_{0}$ are constants. The magnetic separatrix is expressed by the relation $\Phi(r, z)=0$. If $\Phi_{0}=0$, the magnetic separatrix coincides with the plasma-vacuum boundary. If $\Phi_{0}>0$, the plasma can extend outside the magnetic separatrix.

Three types of plasma model are considered in a cylindrical conducting vessl: case (a), where the plasma is confined by a uniform external field and the periodic boundary condition is imposed at two axial edges; case (b), where the plasma is confined by a mirror external field and the fixed boundary condition is imposed at two axial edges; and case (c), where the plasma rotates around the major axis in a uniform field with the axially periodic boundary condition. The initial equilibrium state is obtained by solving the GradShafranov equation by an iteration method. ${ }^{13}$ The initial perturbation is assumed to be

$$
v_{z}=v_{0}\left(p / p_{0}\right)^{(\gamma-1) / \gamma} \cos (n \phi),
$$

so that both the internal and external tilt modes can be induced. Here $n$ is the toroidal mode number, $\phi$ is the azimuthal coordinate, and $v_{0}=10^{-2} v_{A 0}, \quad v_{A 0}$ $=\sqrt{\langle\mathbf{B} \cdot \mathbf{B}\rangle / \rho_{0}} ;\langle F(\mathbf{x})\rangle$ means the volume average of $F(\mathbf{x})$ in the plasma region.

We carry out several simulation runs to obtain appropriate simulation parameters. Almost the same results are obtained from the three runs where the numbers of the grid points along the radial coordinate $n_{r}$ are 33,49 , and 65 , respectively. Figure 3 shows the temporal evolution of the $z$ component of the kinetic energy for the cases where the numbers of the grid points along the azimuthal coordinate $n_{\phi}$ are $4,8,12$, and 16 , respectively. It should be noted in Fig. 3 that the artificial smoothing reduces the growth rate of the

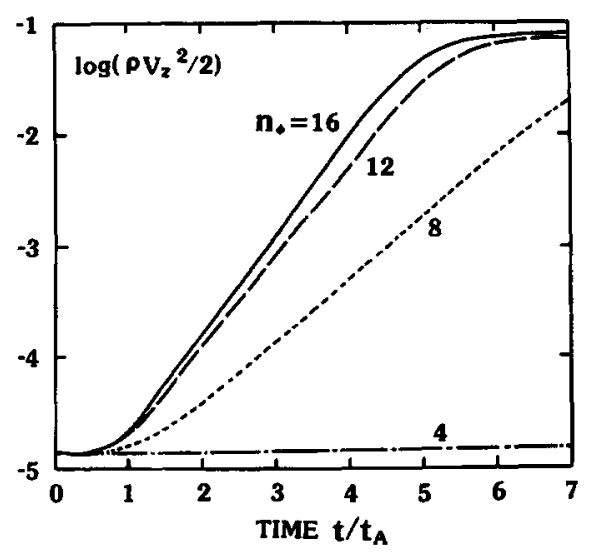

FIG. 3. Temporal evolution of the $z$ component of the kinetic energy for the cases where the number of the grid points along the azimuthal coordinate $n_{\phi}$ is equal to 4 (dotted-dashed line), 8 (dotted line), 12 (dashed line), and 16 (solid line), respectively.

tilt mode and the results look as if the system was stable against the tilt mode for $n_{\phi}=4$ (dotted-dashed line). As $n_{\phi}$ increases, the growth rate becomes larger and approaches the theoretical value. Since the main interest of this paper is in the behavior of the $n=1$ mode, it is sufficient to fix the number of the azimuthal grid points $n_{\phi}$ to 16 . Thus the simulation domain is implemented on a $(65 \times 16 \times 65)$ point grid in cylindrical coordinates $(r, \phi, z)$. The aspect ratio of the simulation cylinder $z_{c} / r_{c}$ is equal to 10 for the prolate FRC and 4 for the oblate and spherical FRC's. The time step is adjusted to satisfy the relation $\alpha \leqslant 1$ everywhere. The dependence of the result on the smoothing frequency is also checked. It is found that the $n=1$ mode evolves almost independently of the smoothing frequency while the high $n$ modes comparable to the grid-sized wavelength tend to be suppressed at low amplitudes as the frequency increases. We carry out the smoothing once at every four time steps in the present simulation.

\section{SIMULATION RESULTS}

\section{A. Uniform field case}

Four simulation runs with different values of $\sigma$ are carried out in the uniform external field. The simulation parameters are given in Table $I$, where $Z_{\text {sp }}$ is the plasma length along the $z$ axis, $R_{\mathrm{sp}}$ is the radial distance of the magnetic separatrix, $\beta_{\mathrm{ex}}$ is the average plasma beta corresponding to the external magnetic field on the midplane, $\Phi_{p}$ is the poloidal magnetic flux in the plasma region, and $\Phi_{e x}$ is the external magnetic flux. The initial configurations of the magnetic field are plotted in Fig. 4. The shape of the magnetic separatrix becomes more prolate and the external beta $\beta_{\mathrm{ex}}$ in-

TABLE I. Simulation parameters.

\begin{tabular}{cccccc}
\hline \hline$\sigma$ & $R_{\text {sp }} / r_{c}$ & $Z_{\text {sp }} / r_{c}$ & $Z_{\text {sp }} / R_{\text {sp }}$ & $\beta_{\text {ex }}$ & $\Phi_{\rho} / \Phi_{\text {ex }}$ \\
\hline 2.1 & 0.96 & 2.69 & 2.80 & 0.56 & 3.92 \\
1.8 & 0.95 & 3.09 & 3.25 & 0.57 & 2.71 \\
1.5 & 0.92 & 3.92 & 4.25 & 0.59 & 1.56 \\
1.2 & 0.87 & 5.26 & 6.02 & 0.62 & 0.81 \\
\hline
\end{tabular}



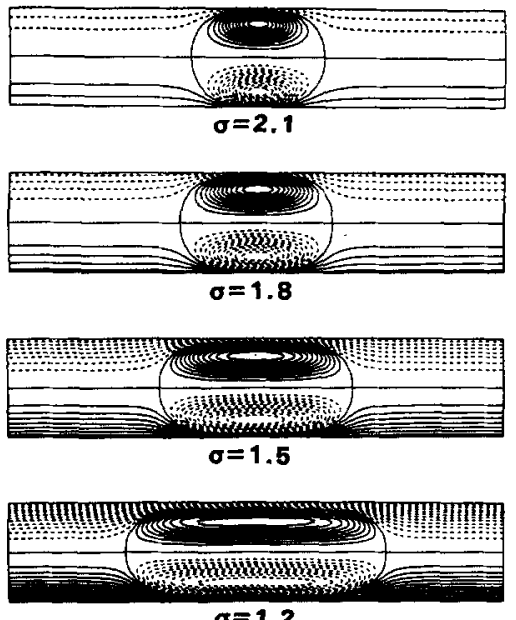

$\sigma=1.2$

FIG. 4. Magnetic field configuration in the poloidal plane for the case where the FRC plasma is confined by the uniform external field. The top, second, third, and bottom panels show the equilibrium configurations with the parameter $\sigma=2.1,1.8,1.5$, and 1.2 , respectively.

creases as the parameter $\sigma$ decreases. It turns out that the value of $\beta_{\mathrm{ex}}$ is nearly equal to the one predicted by the average beta condition, ${ }^{14} \beta_{\mathrm{ex}}=1-\left(R_{\mathrm{sp}} / r_{\mathrm{c}}\right)^{2} / 2$, and that this condition holds more precisely as the plasma length increases. The distance between the separatrix and the wall becomes slightly longer as the poloidal magnetic flux decreases.

Figure 5 shows the vector plots of the magnetic field, plasma velocity, pressure, and the density contours in the poloidal plane $\phi=0$ at $t=0$ (top) and $t=4 t_{\mathrm{A}}$ (bottom) for $\sigma=1.5$. Here $t_{\mathrm{A}}=r_{c} / v_{\mathrm{A} 0}$ is the transit time for the Alfvén wave and the radial scale is magnified by two and onehalf times for convenience. The nonzero plasma velocity along the $z$ axis is given in the plasma region as an initial

$$
\mathbf{t}=\mathbf{0}
$$
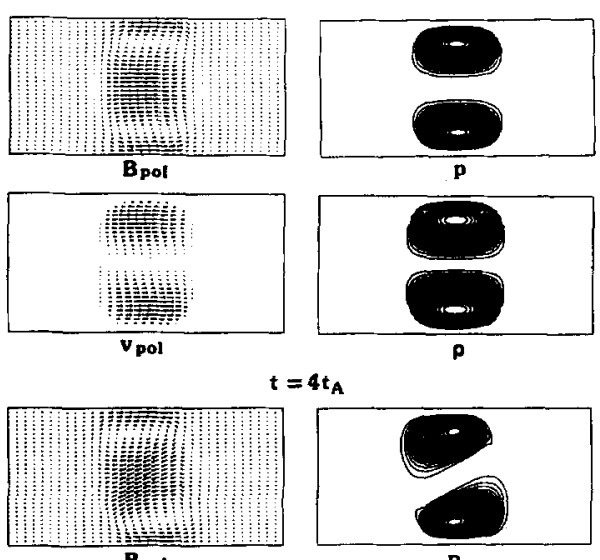

Bpol

$t=4 t_{A}$
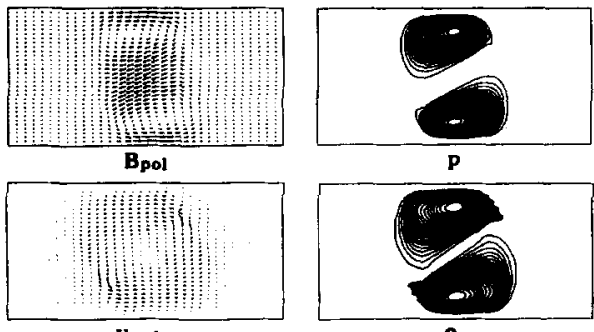

FIG. 5. Magnetic field, matter velocity, thermal pressure, and mass density in the poloidal plane at $t=0$ (top) and $t=4 t_{\mathrm{A}}$ (bottom) for the case of $\sigma=1.5$, where the radial scale is magnified by two and one-half times for convenience. perturbation, as is seen in the top panel of Fig. 5. The initial perturbation induces a circulating flow inside the magnetic separatrix that makes the plasma tilt internally. The internal tilt mode grows as time elapses, and the corresponding changes in the density and pressure profiles are recognized in the bottom of Fig. 5. As we mentioned in the Introduction, there are two severe problems to be overcome in treating the evolution numerically, i.e., the appearance of numerical error at the singular point $r=0$, which sometimes leads to the numerical blowup, and at the plasma-vacuum boundary. Figure 5 indicates that these problems are beautifully cleared in our scheme, and consequently the full nonlinear evolution of the tilting instability is pursued satisfactorily.

In order to examine the global structure of the magnetic field, we introduce a scalar function $\Psi(r, z)$ that describes the configuration of the magnetic field on the poloidal cross section, namely,

$$
\begin{aligned}
\Psi(r, z)= & 2 \pi \int_{r_{u s}}^{r} d r^{\prime} r^{\prime} \\
& \times\left[B_{z}\left(r^{\prime}, \phi_{0}, z\right)-B_{\mathrm{ex}}\right]+2 \pi \int_{0}^{r} d r^{\prime} r^{\prime} B_{\mathrm{ex}},
\end{aligned}
$$

where $\phi_{0}$ is constant. Since the magnetic field is not necessarily axisymmetric in a strict sense except at the initial stage, the function $\Psi$ may not represent the magnetic flux function. The amplitude of the toroidal magnetic field, however, remains far smaller than that of the poloidal component during the simulation. Therefore the function $\Psi$ can be regarded as the function representing the global magnetic field configuration. Figure 6 shows the temporal evolution of the function $\Psi(r, z)$ for the same case as Fig. 5. The initial separatrix takes a racetrack form in the uniform external field. As the internal mode grows, the deformation starts in the region near the X point of the magnetic field $\left(t=3 t_{\mathrm{A}}\right)$. The defor-

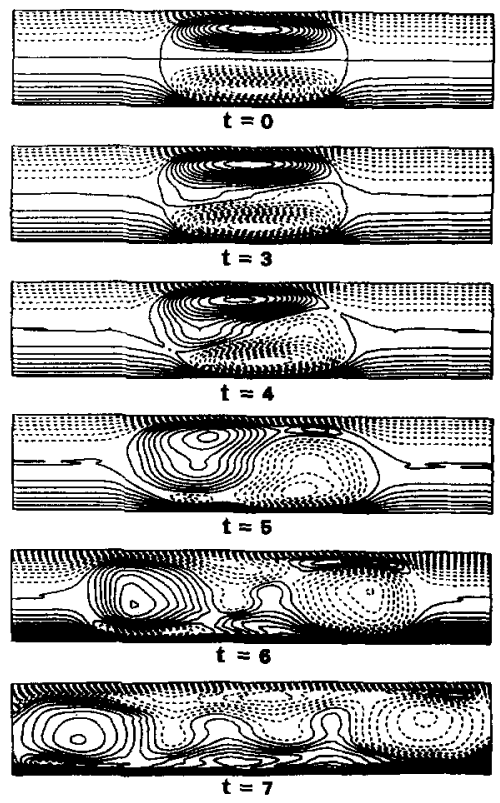

FIG. 6. Temporal evolution of the function $\Psi(r, z)$ for the same case as Fig. 5. Each panel shows the tilt deformation of the magnetic field at $t=0$ (top), $3 t_{\mathrm{A}}$ (second), $4 t_{\mathrm{A}}$ (third), $5 t_{\mathrm{A}}$ (fourth), $6 t_{\mathrm{A}}$ (fifth), and $7 t_{\mathrm{A}}$ (bottom), respectively. 

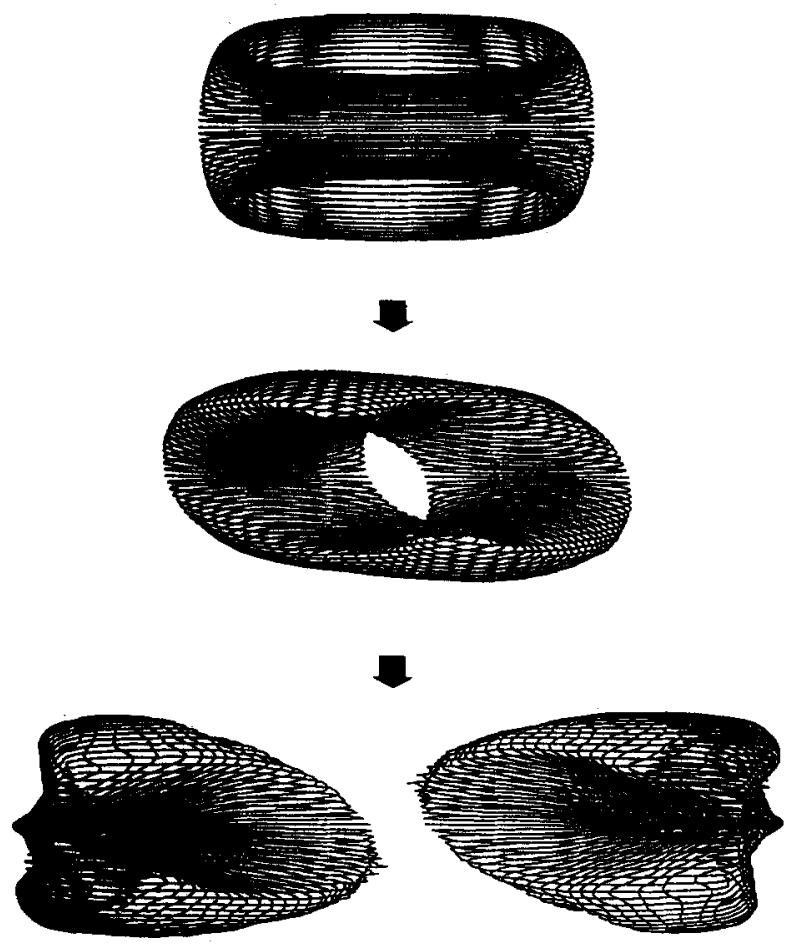

FIG. 7. Three-dimensional display of the pressure contour at $t=0$ (top), $t=5 t_{\mathrm{A}}$ (middle), and $t=6 t_{\mathrm{A}}$ (bottom) for the same case as Fig. 6 .

mation extends gradually to the inner region of the FRC plasma. Finally, the field null point suffers from shifting and the tilting instability is completed internally $\left(t=5 t_{\mathrm{A}}\right)$. The internal deformation gives rise to a pressure imbalance at the plasma-vacuum boundary and triggers an external mode which generates the outgoing flow beyond the boundary $\left(t=5 t_{\mathrm{A}}\right)$. The uniform external field cannot work to stop the plasma motion along the $z$ axis, and thus, the external mode grows until the FRC plasma is torn into two pieces $\left(t=6 t_{\mathrm{A}}\right)$. The pieces move away toward the axial edges. At this stage magnetic reconnection takes place between the plasma field and the external field, and the confinement of the FRC plasma is completely destroyed. ${ }^{15}$ When the plasma flow reaches the edge, the simulation is terminated $\left(t=7 t_{\mathrm{A}}\right)$. The splitting of the FRC plasma is also seen in the pressure distribution. Figure 7 shows a three-dimensional display of the contour maps of the thermal pressure for the same case as Fig. 6. The axisymmetric pressure profile is deformed by the internal tilt mode (middle panel). The plasma flow along the $z$ axis breaks the plasma into two pieces (bottom panel).

Now let us focus our attention on the $\sigma$ dependence of the growth rate of the $n=1$ mode. Figure 8 shows the temporal evolution of the $n=1$ mode for the cases where the parameter $\sigma$ is equal to 1.2 (solid), 1.5 (dashed), 1.8 (dotted), and 2.1 (dotted-dashed line), respectively; and the vertical axis denotes $\left.\log [\mathbf{v} \cdot \mathbf{v}]\right|_{(n=1)}$. The gradient of the growth curve becomes steeper as $\sigma$ increases. This phenomenon is quite understandable when we take into account the fact that the plasma length decreases as $\sigma$ increases (see Table I). That is, the FRC plasma with a shorter plasma length can tilt faster. The growth rate versus the plasma length is plotted in Fig. 9, where the growth rate is defined by the

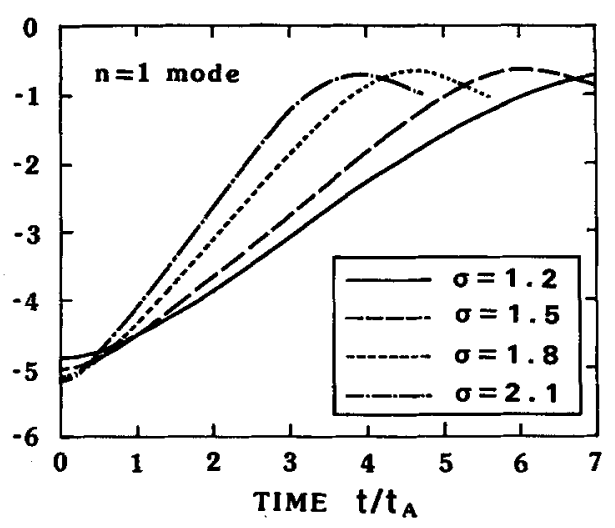

FIG. 8. Temporal evolution of the tilt mode $(n=1)$ in the uniform external field for the cases where the parameter $\sigma$ is equal to 1.2 (solid line), 1.5 (dashed line), 1.8 (dotted line), and 2.1 (dotted-dashed line), respectively. The vertical axis stands for $\left.\log [\mathbf{v} \cdot \mathbf{v}]\right|_{(n=1)}$.

inverse of the $e$-folding time of the mode amplitude and estimated by the gradient in the range of $0.2<t v_{\mathrm{A}} / Z_{\mathrm{sp}}<1.2$. It is noteworthy that the growth rate is in proportion to the inverse of the plasma length and the growth time of the tilting instability is nearly equal to the transit time for the Alfvén wave to propagate over the plasma length, i.e., $t_{\mathrm{grow}}$ $\approx Z_{\mathrm{sp}} / v_{\mathrm{A}}$. These results imply that the tilting instability is essentially ideal and the resistive process is secondary. The reason for this is that there is no toroidal field in the FRC plasma and the poloidal flux tubes are not magnetically linked with each other, so that the reconnection process cannot necessarily be involved in the tilting instability and the resulting splitting phenomena.

\section{B. Mirror field case}

In order to examine the suppression effect of the tilting instability by adding the pinching magnetic field at both ends of the FRC plasma, we carry out simulation runs in the mirror field, which is described by the poloidal flux function

$$
\Phi_{m}(r, z)=\Phi_{m 0} r J_{1}(k r) \cosh (k z) .
$$

Here $J_{n}(x)$ is the Bessel function of order $n$ and $\Phi_{m 0}$ and $k$

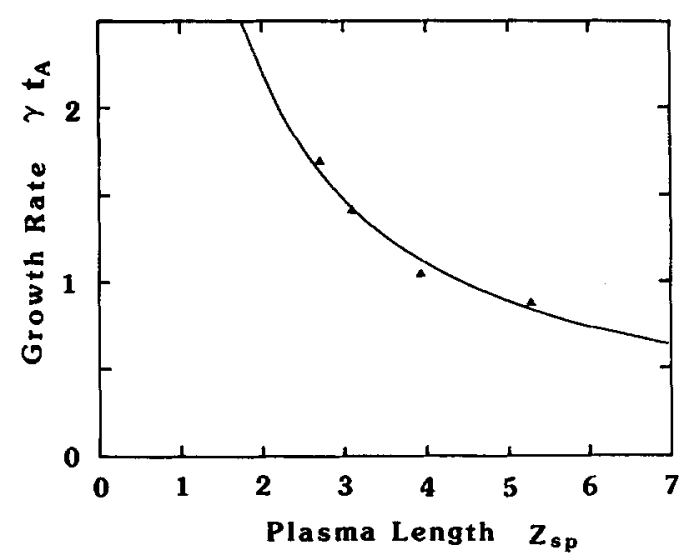

FIG. 9. Growth rate versus plasma length for the same case as Fig. 8 where the closed triangles show the simulation results and the dotted line shows the relation $\gamma t_{\mathrm{A}} \propto 1 / Z_{\mathrm{sp}}$. Notice that the growth rate is in proportion to the inverse of the plasma length. 
TABLE II. Simulation parameters.

\begin{tabular}{cccccc}
\hline \hline$R_{m}$ & $R_{\mathrm{sp}} / r_{\mathrm{c}}$ & $Z_{\mathrm{sp}} / r_{\mathrm{c}}$ & $Z_{\mathrm{sp}} / R_{\mathrm{sp}}$ & $\beta_{\mathrm{ex}}$ & $\Phi_{p} / \Phi_{\mathrm{ex}}$ \\
\hline 1.0 & 0.92 & 4.48 & 4.85 & 0.59 & 1.63 \\
1.54 & 0.93 & 4.22 & 4.54 & 0.57 & 1.78 \\
3.76 & 0.94 & 3.76 & 4.02 & 0.60 & 2.05 \\
6.13 & 0.94 & 3.56 & 3.78 & 0.58 & 2.21 \\
$6.85^{\mathrm{a}}$ & 0.94 & 1.88 & 2.01 & 0.68 & 2.00 \\
$20.9^{\mathrm{a}}$ & 0.94 & 1.48 & 1.57 & 0.71 & 2.33 \\
\hline
\end{tabular}

${ }^{2}$ Note that the aspect ratio of the simulation cylinder is fixed to 4 for the last two runs.

are constants. The simulation parameters are given in Table II, where $R_{m}=B_{z}$ (edge) $/ B_{z}$ (center) is the mirror ratio of the external field, and the external magnetic flux $\Phi_{\text {ex }}$ is given by the value on the midplane. The initial configurations of the magnetic field are shown in Fig. 10 for the cases where the mirror ratio $R_{m}$ is equal to 1.0 (top), 1.54 (second), 3.76 (third), and 6.13 (bottom diagram), respectively. The parameter $\sigma$ is fixed to 1.5 for every case. The shape of the magnetic separatrix becomes less prolate as the mirror ratio increases. Figure 11 shows the temporal evolution of the pressure distribution in the poloidal plane for $R_{m}=6.13$. The initial axisymmetric profile is deformed gradually as the internal tilt mode grows $\left(t=3,4 t_{\mathrm{A}}\right)$. When the internal mode grows sufficiently, the external mode sets in $\left(t=5 t_{\mathrm{A}}\right)$. Finally, the FRC plasma is broken into two pieces by the outgoing flow $\left(t=6 t_{\mathrm{A}}\right)$. In this way, the disruption process of the FRC plasma is considered the same as that for the uniform field case.

The time history of the $n=1$ mode is plotted in Fig. 12 for the cases where $R_{m}=1.0$ (solid), 1.54 (dashed), 3.76 (dotted), and 6.13 (dotted-dashed line), respectively. The time in Fig. 12 is normalized by the Alfvén transit time $Z_{\mathrm{sp}} / v_{\mathrm{A}}$. As can be seen in Fig. 12, the growth rate decreases slightly as the mirror ratio increases. The growth time is nearly equal to Alfvén transit time, which is the same as that for the uniform field case. Since the velocity of the outgoing

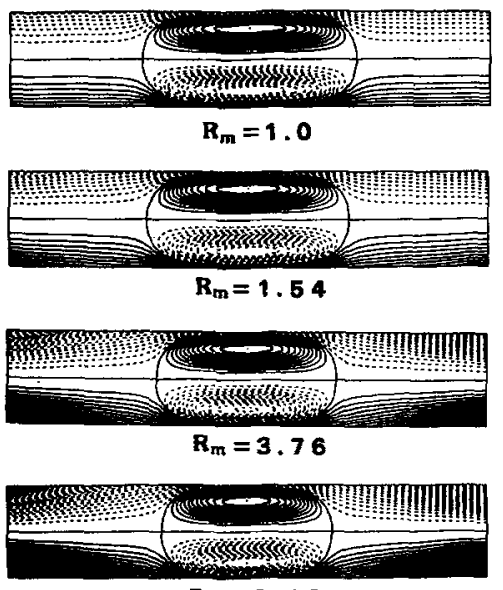

$R_{m}=6.13$

FIG. 10. Magnetic field configuration in the poloidal plane for the case where the FRC plasma is confined by the mirror field. The top, second, third, and bottom panels show the equilibrium configurations of the parameter $R_{m}=1.0,1.54,3.76$, and 6.13 , respectively.
PRESSURE ( $R_{m}=6.13$ )

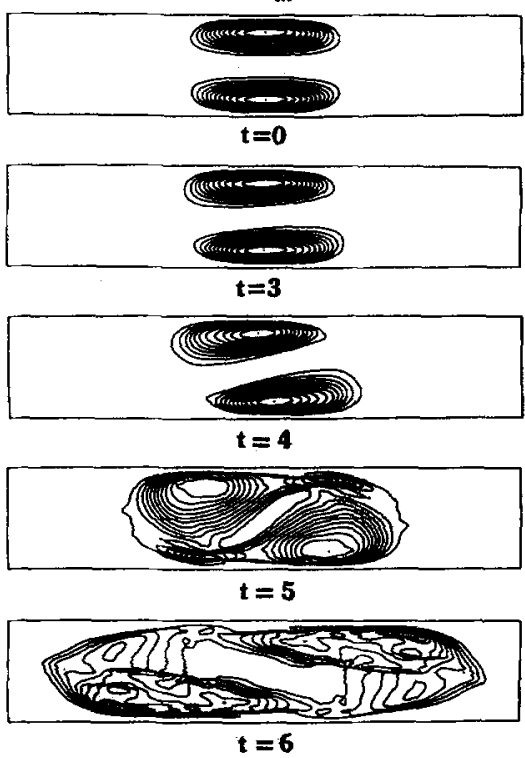

FIG. 11. The pressure profile in the poloidal plane at $t=0(\mathrm{top}), 3 t_{\mathrm{A}}$ ( $\mathrm{sec}$ ond), $4 t_{\mathrm{A}}$ (third), $5 t_{\mathrm{A}}$ (fourth), and $6 t_{\mathrm{A}}$ (bottom) for the case of $R_{m}$ $=6.13$.

flow toward the axial edge is comparable to the Alfvern velocity, the external mirror field is needed to have the radial component comparable to the dynamical pressure of the outgoing flow just at the outside of the magnetic separatrix in order to suppress the external mode.

\section{Spin-stabilization effect}

It is reported ${ }^{16}$ that the lifetime of a spheromak against the tilting instability was made several times longer by spinning the plasma with an Alfvén Mach number of $M_{\mathrm{A}}>1$. Let us examine the spin-stabilization effect against the tilting instability of the FRC plasma. The initial condition is obtained by gradually accelerating the rotation inside the magnetic separatrix with the use of a two-dimensional simulation code. ${ }^{16}$ The angular velocity of the rotation is assumed

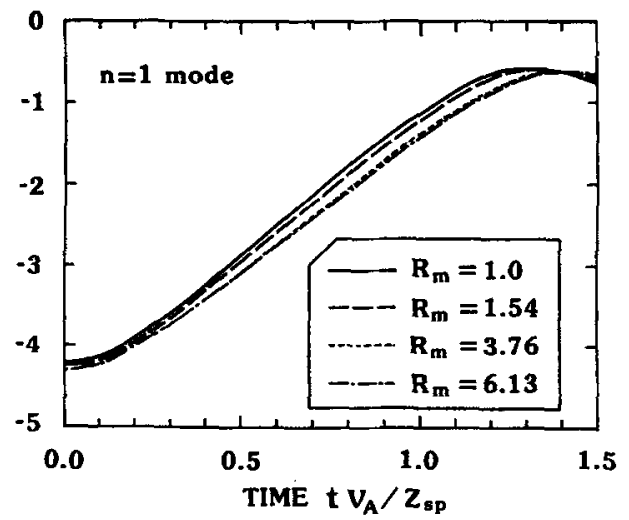

FIG. 12. Temporal evolution of the tilt mode $(n=1)$ in the mirror field for the cases where the parameter $R_{m}$ is equal to 1.0 (solid line), 1.54 (dashed line), 3.76 (dotted line), and 6.13 (dotted-dashed line), respectively. The vertical axis denotes $\left.\log [v \cdot v]\right|_{(n=1)}$ and the time is normalized by the Alfvén transit time $Z_{\mathrm{sp}} / v_{\mathrm{A}}$. 
TABLE III. Simulation parameters.

\begin{tabular}{cccccc}
\hline \hline$M$ & $R_{\mathrm{sp}} / r_{\mathrm{c}}$ & $Z_{\mathrm{sp}} / r_{c}$ & $Z_{\mathrm{sp}} / R_{\mathrm{sp}}$ & $\beta_{\mathrm{ex}}$ & $\Phi_{p} / \Phi_{\mathrm{cx}}$ \\
\hline 0.0 & 0.84 & 5.62 & 6.69 & 0.69 & 0.56 \\
0.5 & 0.83 & 5.67 & 6.86 & 0.64 & 0.52 \\
1.0 & 0.85 & 5.32 & 6.27 & 0.51 & 0.52 \\
1.5 & 0.88 & 5.31 & 6.02 & 0.37 & 0.52 \\
\hline
\end{tabular}

to be in proportion to the poloidal flux function, i.e., $\Delta \Omega=\Omega_{*} \Phi(r, z)$. For technical reasons we chose a nonrotating equilibrium with plasma leakage beyond the separatrix as the starting configuration, i.e., $\Phi_{0}=-0.3 \Phi_{\mathrm{nI}}$. An artificial viscosity is added in the toroidal component of the induction equation to approach a solution satisfying the relations $B_{\phi}=0$ and $\Omega=\Omega(\Phi)$ as quickly as possible.

Four simulation runs are carried out in order to examine the Mach number dependence on the tilting instability. The simulation parameters are given in Table III. The field null point tends to shift outward as the Mach number becomes larger, because of the centrifugal force. Figure 13 shows the temporal evolution of the pressure distribution in the poloidal plane for the nonrotating case (left panel) and also for the rotating case (right panel) where the Mach number $M$ ( $=v_{\phi} / \sqrt{\gamma P / \rho}$ ) is equal to 1.5. It is clear that for the nonrotating case the pressure profile is deformed by the internal tilt mode and the plasma is broken into two pieces in a manner similar to the cases discussed in Sec. III A. When the plasma spins with $M=1.5$, the pressure profile is also slightly deformed, which indicates that the instability is largely weakened.

Let us roughly estimate the threshold value of the spinning Mach number leading to the stabilization of the tilt mode. The outward transfer of angular momentum is needed for the rotating plasma to shift inward as a result of the tilt deformation. When there is no toroidal magnetic field, namely, no magnetic tension, angular momentum cannot be transferred in the axisymmetric equilibrium configuration. Suppose that a small piece of plasma moves slightly inward by $\delta r$. Then, the piece is forced to move to the original position by the centrifugal force unless any deceleration occurs. In this case, however, the toroidal magnetic field is generated by the induction electric field $\delta r \Omega \times B_{\mathrm{pol}}$ and the magnetic tension due to the poloidal and the induced toroidal fields acts to decelerate the rotating plasma, thus accelerating the tilt deformation. Rough estimation leads to the result that the restoring centrifugal force overcomes the magnetic tension when $\delta r B_{\mathrm{pol}} B_{\mathrm{ex}} / r^{2}<\rho \delta r \Omega^{2}$. Because the relations $B_{\mathrm{pol}}^{2} / 2+p \approx B_{\mathrm{ex}}^{2} / 2$ and $2 p / B_{\mathrm{ex}}^{2} \approx 0.5$ (see Tables I-III) hold in the FRC plasma, the condition of $M>1.3$ is needed for the spin stabilization of the tilting instability.

Let us examine the dependence of the growth rate on the spinning Mach number in more detail. The growth of the tilting instability is drawn in Fig. 14 for the cases where the Mach number on the field null point $M$ is equal to 0.0 (solid), 0.5 (dashed), 1.0 (dotted), and 1.5 (dotted-dashed line), respectively. Notice that the tilt mode also grows slowly for $M=1.5$, though the deformation of the pressure profile stays at a low level (see Fig. 13). This result implies that the FRC plasma is not stable against the tilting instability even if the spin is as high as $M=1.5$. The growth rate versus the Mach number is plotted in Fig. 15 for the same case as Fig. 14. It is worth noting that the growth rate decreases linearly as the Mach number increases. The dotted line shows the relation $\gamma v_{\mathrm{A}} / Z_{\mathrm{sp}}=-2.2 \mathrm{M}+5.7$. Figure 15 leads us to a conclusion that the tilt disruption can be suppressed by driving the plasma to spin with such a high veloc-
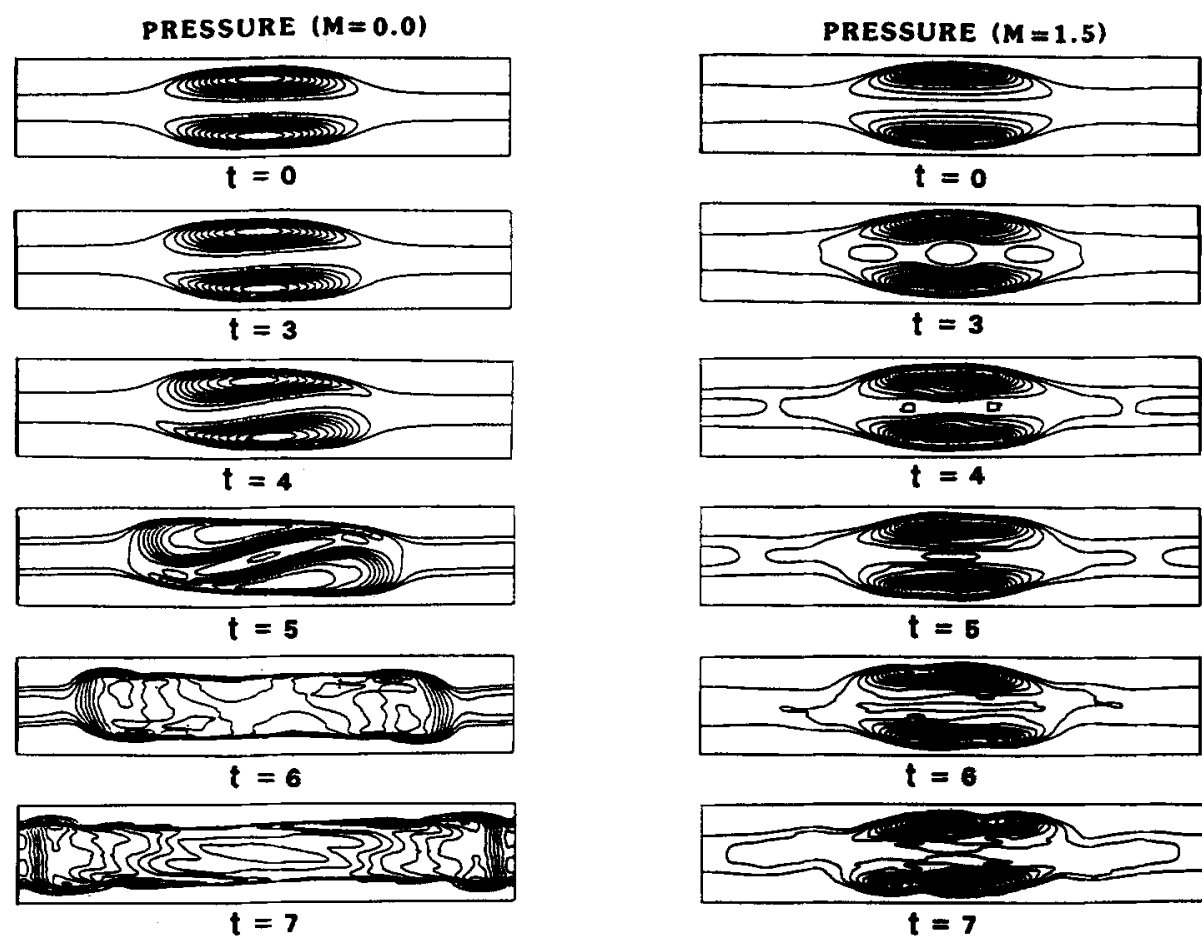

FIG. 13. Temporal evolutions of the pressure profiles in the poloidal plane for the nonrotating case (left panels) and for the case of the spinning Mach number $M=1.5$ (right panels), where each panel shows the configuration at six different periods $(t=0,3,4,5,6$, $\left.7 t_{\mathrm{A}}\right)$. Notice that the FRC plasma is disrupted by the tilt mode for the nonrotating case while the tilt deformation scarcely appears for the rapidly rotating case. 


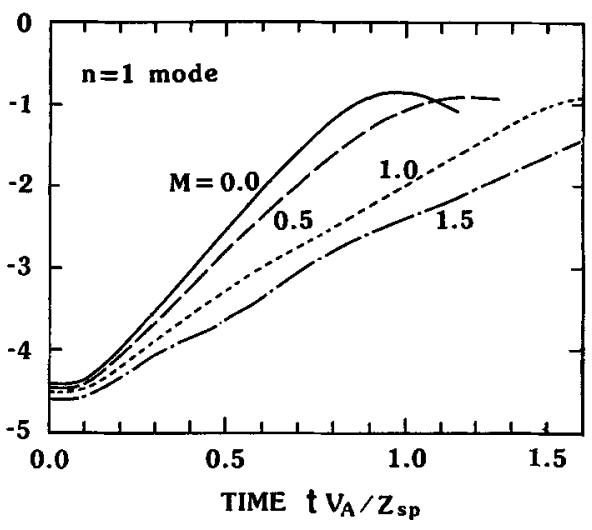

FIG. 14. The growth curves of the tilting instability for the cases where the Mach number on the field null point $M$ is equal to 0.0 (solid line), 0.5 (dashed line), 1.0 (dotted line), and 1.5 (dotted-dashed line), respectively. The vertical axis represents $\left.\log \left[\mathbf{v}_{z} \cdot \mathbf{v}_{z}\right]\right|_{(n=1)}$.

ity as $M>2.5$. This threshold value is somewhat larger than the value reported by Milroy et al. ${ }^{15}$

\section{Oblate FRC}

According to the linear analysis ${ }^{1-3}$ the FRC plasma becomes stable against the internal tilt mode when the shape of the magnetic separatrix becomes oblate, i.e., $Z_{\mathrm{sp}} / 2 R_{\mathrm{sp}}<1.0$. In this subsection we shall investigate the nonlinear evolution of the tilting instability in the oblate FRC plasma confined by a mirror external field. Two types of the simulation model are considered in the cylindrical vessel with the aspect ratio $z_{c} / r_{c}=4$, i.e., the spherical FRC of the plasma aspect ratio $Z_{\mathrm{sp}} / 2 R_{\mathrm{sp}}=1.0$ and the oblate $\mathrm{FRC}$ of $Z_{\mathrm{sp}} / 2 R_{\mathrm{sp}}$ $=0.78$. The physical parameters are listed in the last two lines in Table II. The initial configuration of the magnetic field in the poloidal plane is illustrated in Fig. 16 for the two cases. A strong mirror field of $R_{m}=20.9$ is adopted to obtain the initial equilibrium solution of the oblate FRC.

Figure 17 shows the tilt deformation of the pressure profile in the poloidal plane $\phi=0$ for the oblate case. It is noteworthy that there is no clear deformation of the pressure distribution associated with the internal tilt mode, but the

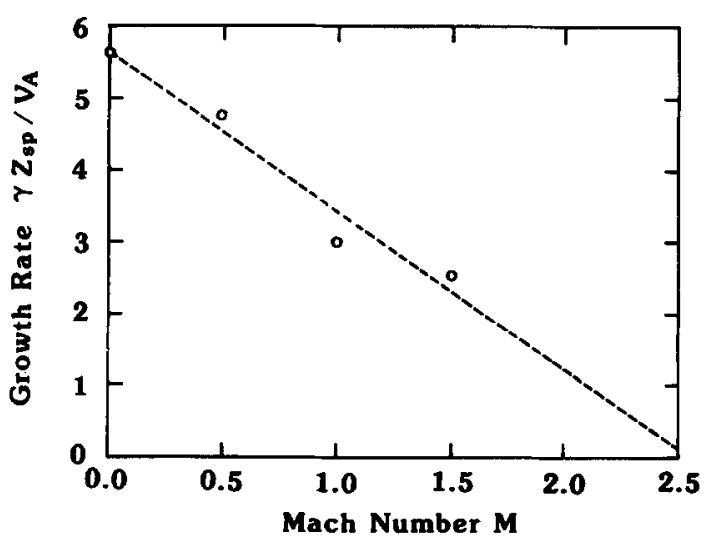

FIG. 15. Growth rate versus Mach number for the same case as Fig. 14 where the open circles show the simulation results and the dotted line represents the relation $w_{\mathrm{A}} / Z_{\mathrm{sp}}=-2.2 M+5.7$. It is noteworthy that the growth rate decreases in proportion to the Mach number.

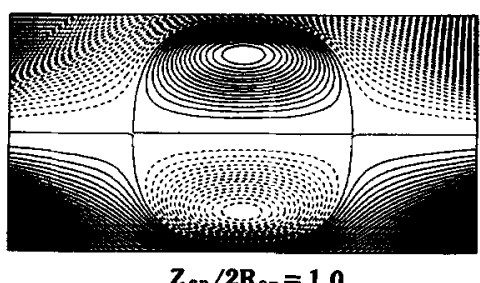

$Z_{\mathrm{sp}} / 2 \mathrm{R}_{\mathrm{sp}}=1.0$

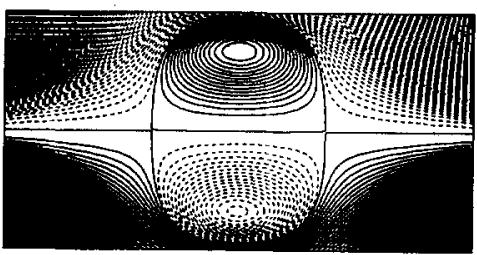

$Z_{\mathrm{sp}} / 2 R_{\mathrm{sp}}=0.78$

FIG. 16. The initial configuration of the magnetic field for the spherical FRC (top) and the oblate FRC (bottom) in the mirror field.

plasma is deformed directly by the external mode, ${ }^{17}$ which induces the plasma flow and changes the plasma-vacuum boundary shape (see the middle panel of Fig. 17). This result is consistent with the linear theory that the oblate FRC plasma is stable against the internal tilt mode. The plasma tilts freely toward the region where the magnetic energy density is at a minimum in the initial configuration (Fig. 16). Finally, a standing shock is formed by the interaction between the tilted plasma and the mirror field $\left(t=4 t_{\mathrm{A}}\right)$. It is concluded therefore that an oblate FRC in the mirror field can be disrupted by the development of an external tilt mode. For the spherical case we obtain almost the same result as that for the oblate case.

We plot the dependence of the growth rate $\gamma Z_{\mathrm{sp}} / v_{\mathrm{A}}$ on the plasma aspect ratio $Z_{\mathrm{sp}} / 2 R_{\mathrm{sp}}$ in Fig. 18, together with the results of the uniform field case (open triangles) and the mirror field case (open circles). The growth rate is almost independent of the plasma aspect ratio for the uniform field case, while for the mirror field case the growth rate decreases

Pressure $\left(Z_{\text {sp }} / 2 R_{s p}=0.78\right)$

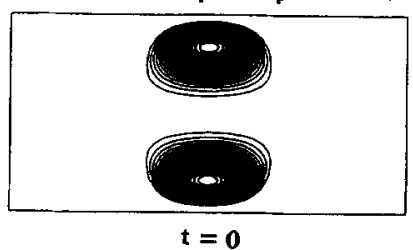

$\mathbf{t}=\mathbf{0}$

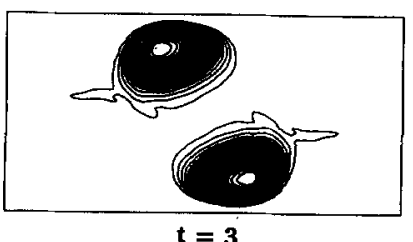

$\mathbf{t}=\mathbf{3}$

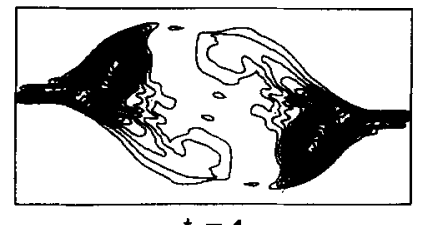

FIG. 17. The pressure profile in the poloidal plane at $t=0$ (top), $3 t_{\mathrm{A}}$ (middle), and $4 t_{\mathrm{A}}$ (bottom) for the case of $Z_{\mathrm{sp}} / 2 R_{\mathrm{sp}}=0.78$. Standing shock appears in the bottom diagram where the tilted plasma encounters the strong mirror field. 


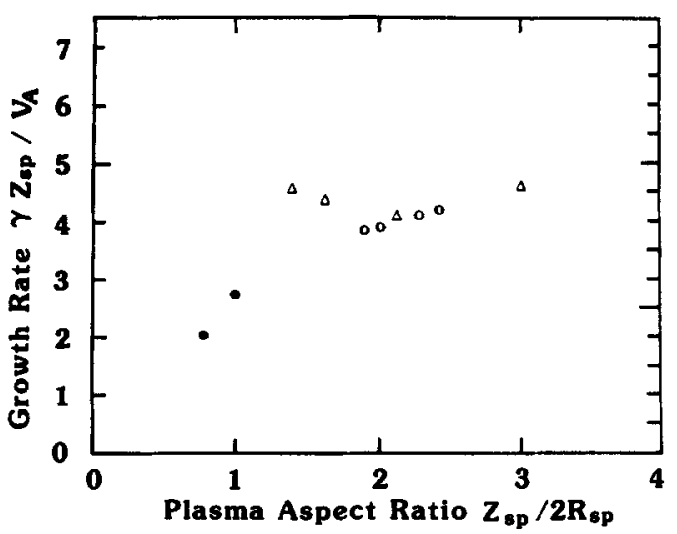

FIG. 18. Growth rate versus plasma aspect ratio for the uniform field cases (open triangles) and for the mirror field cases where the open circles show the results of the prolate FRC and the closed circles show the results of the spherical FRC and the oblate FRC.

as the shape of the magnetic separatrix becomes more oblate. It is concluded that, though the growth rate decreases compared with the prolate FRC, the oblate plasma is unstable against the external tilt mode.

\section{DISCUSSION}

We developed a new simulation code with a fourth-order accuracy both in space and time and applied the code to a FRC plasma confined in a conducting cylindrical vessel. This simulation code succeeded in describing the nonlinear evolution of the tilting instability with sufficient reliance. The results are summarized as follows.

(i) The prolate FRC plasma is unstable against an internal tilt mode both for the case where the plasma is confined by a uniform external field and for the case where the plasma is confined by a mirror field. When the plasma suffers from a sufficient deformation, the pressure imbalance triggers an external mode at the plasma-vacuum boundary. Finally, the FRC plasma is broken into two pieces by the outgoing flows along the major axis.

(ii) The growth time of the internal tilt mode is given by the transit time for the Alfvén wave to propagate over the plasma length. This result implies that the tilting instability is essentially ideal.

(iii) The plasma spin around the major axis results in the decrease of the growth rate of the tilting instability. If we can drive the plasma to rotate with the spinning Mach number $M>2.5$, the prolate FRC plasma can be stabilized against the tilt mode.

(iv) The FRC equilibrium with an oblate shape is obtained by adding a large pinching field at the axial edges. In contrast to the prolate FRC, the oblate FRC plasma becomes unstable against the external tilt mode.

Before concluding the paper let us discuss the applicability of the model we used. In our model there is the simple relationship between the pressure and the poloidal flux function. The magnetic separatrix is close to the conducting wall in the equilibrium where the plasma is fully confined in the magnetic separatrix. When the plasma leakage is added beyond the separatrix in the equilibrium, the separatrix shifts inward and the value of $R_{\mathrm{sp}} / r_{c}$ approaches the experimental one. ${ }^{4}$ As was reported for the case of $M=0$ in Sec. III C, the plasma leakage did not give rise to any significant change in the simulation results. This result is understandable when we take into account the fact that the tilting instability is triggered by the internal mode in the prolate FRC.

In general, the equilibrium profile of the pressure is given by an arbitrary function of the poloidal flux function $\Phi$ and there are infinite sets of the equilibrium solutions. Thus, from a limited number of simulation runs, we cannot obtain a definite conclusion that any FRC configuration is unstable against the tilt mode. Judging from the linear analysis and the simulation study, however, it seems that the FRC plasma is not in a minimum energy state in a magnetohydrodynamic sense. This may partly be due to the fact that there is no global constraint like the magnetic helicity in the reversed field pinch. ${ }^{18}$

We used a simple spatially uniform resistivity model. There may be an argument that the spatial dependence of the resistivity should be considered. However, the resistivity profile would not lead to an appreciable alteration of the simulation results since the tilting instability is essentially ideal.

The MHD analyses have shown that the FRC plasma is unstable against the tilt mode except in a highly spinning FRC. The observational fact that there is no experimental evidence of tilt disruption may be explained by taking the ion kinetic effect into account. In order to clarify the whole feature of the tilting instability, it is necessary to investigate the nonlinear evolution by using a special particle simulation code that can deal with a MHD time scale.

\section{ACKNOWLEDGMENTS}

We are grateful to $K$. Nishikawa for his continuous interest in this work and T. Hayashi for his useful suggestions.

This work is supported by a Grant-in-Aid of the Ministry of Education, Science, and Culture in Japan.

'W. N. Rosenbluth and M. N. Bussac, Nucl. Fusion 19, 489 (1979); J. H. Hammer, Nucl. Fusion 21, 488 (1981).

${ }^{2}$ J. L. Schwarzmeier, D. C. Barnes, D. W. Hewett, C. E. Seyler, A. I. Shestakov, and R. L. Spencer, Phys. Fluids 26, 1295 (1983).

${ }^{3}$ R. A. Clemente and C. E. Grillo, Phys. Fluids 27, 658 (1984).

${ }^{4}$ For example, A. G. Eskov, R. Kh. Kurtmullaev, A. P. Kreshchuk, Ya. N. Laukhin, A. I. Malyutin, A. I. Markin, Yu. S. Martyushov, B. N. Mironov, M. M. Orlov, A. P. Proshletsov, V. N. Semenov, and Yu. B. Sosunov, in Plasma Physics and Controlled Nuclear Fusion Research, 1978 (IAEA, Vienna, 1979), Vol. 2, p. 187; W. T. Armstrong, D. G. Harding, E. A. Crawford, and A. L. Hoffman, Phys. Fluids 25, 2121 (1982); S. Ohi, T. Minato, Y. Kawakami, M. Tanjyo, S. Okada, Y. Ito, M. Kako, S. Goto, T. Ishimura, and H. Ito, Phys. Rev. Lett. 51, 1042 (1983); A. L. Hoffman, J. T. Slough, and D. G. Harding, Phys. Fluids 26, 1626 (1983).

${ }^{5}$ D. C. Barnes, J. L. Schwarzmeier, H. R. Lewis, and C. E. Seyler, Phys. Fluids 29, 2616 (1986)

${ }^{6}$ A. Ishida, H. Momota, and L. C. Steinhauer, Phys. Fluids. 31, 3024 (1988).

'H. L. Berk, H. Momota, and T. Tajima, Phys. Fluids 30, 3548 (1987).

${ }^{8}$ R. Horiuchi and T. Sato, Phys. Fluids 31, 1142 (1988); T. Hayashi and T. Sato, Phys. Fluids 28, 3654 (1985). 
${ }^{9}$ W. H. Press, B. P. Flannery, S. A. Teukolsky, and W. T. Vetterling, in Numerical Recipes (Cambridge U.P., Cambridge, 1987), p. 547.

${ }^{10}$ D. Potter, Computational Physics (Wiley, New York, 1980), p. 67.

"R. Courant, K. O. Friedrichs, and H. Lewy, Math. Ann. 100, 32 (1928).

${ }^{12}$ For example, D. D. Schnack, D. C. Baxter, and E. J. Caramana, J. Comput. Phys. 55, 485 (1984).

${ }^{13}$ J. L. Johnson, H. E. Dalhed, J. M. Greene, R. C. Grimm, Y. Y. Hsieh, S. C. Jardin, J. Manickam, M. Okabayashi, R. G. Storer, A. M. M. Todd, D. E. Voss, and K. E. Weimer, J. Comput. Phys. 32, 212 (1979).
${ }^{14}$ W. T. Armstrong, R. K. Linford, J. Lipson, D. A. Platts, and E. G. Sherwood, Phys. Fluids 24, 2068 (1981).

${ }^{15}$ R. D. Milroy, D. C. Barnes, R. C. Bishop, and D. D. Schnack, in Proceedings of the 12th Conference on the Numerical Simulation of Plasmas (Lawrence Livermore National Laboratory, Livermore, CA, 1987), Abstract PM21.

${ }^{16}$ T. Hayashi and T. Sato, J. Phys. Soc. Jpn. 56, 2039 (1987).

${ }^{17}$ C. B. Ruchi and R. V. Lovelace, Phys. Fluids 27, 1789 (1984).

${ }^{18}$ J. B. Taylor, Phys. Rev. Lett. 33, 1139 (1974). 